\title{
RF OPTIONS FOR THE NSNS
}

BNL/NSNS TECHNICAL NOTE

NO. 009

M. Blaskiewicz, J. M. Brennan and Y. Y. Lee

December 5, 1996

ALTERNATING GRADIENT SYNCHROTRON DEPARTMENT BROOKHAVEN NATIONAL LABORATORY UPTON, NEW YORK 11973 


\title{
Rf Options for the NSNS
}

\author{
M. Blaskiewicz, J.M. Brennan, Y.Y. Lee
}

December 5, 1996

\section{Abstract}

Rf options for the NSNS are considered. The strengths and weaknesses of single and dual frequency conventional rf systems, and a barrier cavity rf system are explored. It is found that a two cavity conventional rf system running with harmonics one and two is a substantial improvement over a conventional $h=1$ system. A barrier cavity system is even better, but may require three rf cavities.

\section{Introduction}

The National Spallation Neutron Source includes an accumulator ring with a circumference of $C=$ $208.6 \mathrm{~m}$ that is designed to accumulate $1 \times 10^{14}, 1 \mathrm{GeV}$ kinetic energy protons, via charge exchange injection of $\mathrm{H}^{-}$, in $1 \mathrm{~ms}$. After the beam is accumulated it will be extracted in one turn using a kicker magnet. A $280 \mathrm{~ns}$ gap is required to allow for the kicker rise time. In the early design stages of this ring a radio frequency system running with harmonic number $h=1$ was a natural assumption. However, since uncontrolled losses in the NSNS ring must be kept below $0.01 \%$, it is natural to investigate the possibility of reducing losses via an appropriate choice of rf system. The low losses, coupled with the large beam current, argue that the bunching factor $B_{f}=I_{\text {avg }} / I_{\text {peak }}$, be kept as large as possible. A standard technique for increasing the bunching factor is the addition of a higher harmonic cavity or, more recently, a barrier cavity rf system. This note is concerned with analyzing the strengths and weaknesses of these contending systems.

\section{Contending rf systems}

The present NSNS lattice has four, five meter straight sections which could be used for rf, and a peak voltage of $\approx 40 \mathrm{kV} /$ cavity is a practical design limit. Of the four available straight sections we assume that two or three will go to rf, while the rest are left free for other (possibly unforseen) uses. With these constraints a traditional rf system with one cavity running at $h=1$ and one more, running at a higher harmonic, is feasible. The possibilities of having the higher harmonic running at $h=2$ and $h=3$ have been considered.

Another alternative is to use a barrier cavity rf system. In a barrier cavity system the rf waveform is periodic at the revolution frequency, but contains many Fourier components. The purpose of such a system is to contain the beam in a flat bottomed potential with steeply rising edges. To our knowledge 


\begin{tabular}{|c|c|c|c|c|c|}
\hline case & $\begin{array}{c}\text { voltage } \\
h=1 \\
\mathrm{kV}\end{array}$ & $\begin{array}{c}\text { voltage } \\
h=2 \\
\mathrm{kV}\end{array}$ & $\begin{array}{c}\text { voltage } \\
h=3 \\
\mathrm{kV}\end{array}$ & $\begin{array}{c}\text { voltage } \\
\mathrm{h=1.85} \\
\mathrm{kV}\end{array}$ & $\begin{array}{c}\text { bucket } \\
\text { area } \\
\mathrm{eV}-\mathrm{sec}\end{array}$ \\
\hline 1 & 37.15 & 0 & 0 & 0 & 15.5 \\
\hline 2 & 40.34 & 20.17 & 0 & 0 & 18.6 \\
\hline 3 & 32.56 & 0 & 10.85 & 0 & 13.0 \\
\hline barrier & 0 & 0 & 0 & 116.13 & 25.5 \\
\hline
\end{tabular}

Table 1: rf parameters for contending rf systems.

there are two techniques for generating the necessary rf waveform. The first technique involves several rf systems operating at different harmonic numbers with at least one rf cavity and amplifier for each Fourier harmonic in the waveform. A second technique involves driving the rf cavities in a nonresonant mode $[1,2,3,4]$. In the second case, the basic shape of the current pulse can be found by modeling the cavity as a parallel RLC circuit [3]. The voltage across each circuit element is the same, and the total current through the cavity is the sum of the currents in each element,

$$
I(t)=\frac{V(t)}{R}+\frac{1}{L} \int_{0}^{t} V\left(t^{\prime}\right) d t^{\prime}+C \frac{d V(t)}{d t} .
$$

For an isolated sinewave

$$
V(t)= \begin{cases}V_{0} \sin (\omega t) & 0<\omega t<2 \pi \\ 0 & \text { otherwise }\end{cases}
$$

the required current is

$$
I(t)=\frac{V_{0} \sin (\omega t)}{R}+\frac{V_{0}}{\omega L}+V_{0} \cos (\omega t)\left(\omega C-\frac{1}{\omega L}\right)
$$

when $0<\omega t<2 \pi$ and vanishes otherwise.

For comparison purposes assume a $10 \mathrm{eV}$-sec longitudinal emittance with a $515 \mathrm{~ns}$ bunch length. Additionally, the amplitude and phase of the higher harmonic in the traditional rf systems is set so that the small amplitude synchrotron frequency vanishes. The rf parameters for the four options are shown in Table 1. Note that the non-integer harmonic number associated with the barrier cavity is simply $\omega / \omega_{0}$.

As is clear from the table, the largest bucket area is obtained for the barrier cavity, while case 3 has the smallest bucket area. The reduced bucket area in case 3 is due to the fact that both the rf voltage and its time derivative vanish at the unstable fixed point. We see no reason to consider case 3 further.

Figure 1 shows the phase space for the three remaining alternatives. As is clear from the figure, the dual frequency system has a larger bucket area and a smaller energy spread than the single frequency system, while the barrier cavity system has the largest bucket area and smallest energy spread. At some level these differences are illusory, since $2 \mathrm{rf}$ stations at $h=1$ could be implemented and the barrier cavity voltage used in the figure might require three cavities. However, the ratio of the bunch 


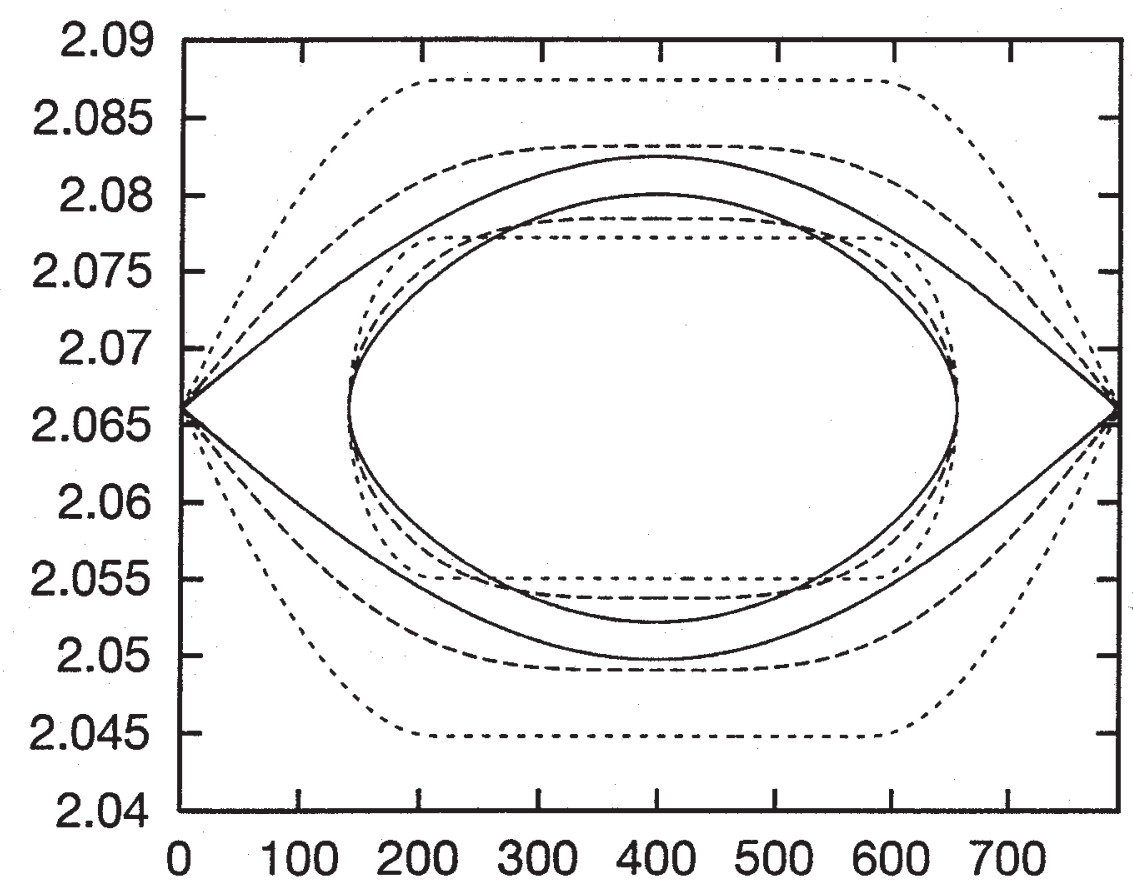

Figure 1: $\gamma$ versus time (ns) for rf buckets and $10 \mathrm{eV}$-sec contours with three rf options: single frequency at $h=1$, solid line, bucket area $=15.5 \mathrm{eV}$-sec;

dual frequency with $h=1$ and $h=2$, long dash, bucket area $=18.6 \mathrm{eV}$-sec; barrier cavity with $h=1.85$, short dash, bucket area $=25.5 \mathrm{eV}$-sec.

\begin{tabular}{|c|c|c|c|}
\hline parameter & $\begin{array}{c}\text { single } \\
\text { frequency }\end{array}$ & $\begin{array}{c}\text { dual } \\
\text { frequency }\end{array}$ & $\begin{array}{c}\text { barrier } \\
\text { cavity }\end{array}$ \\
\hline$C$ & $208.558 \mathrm{~m}$ & $208.558 \mathrm{~m}$ & 208.558 \\
\hline$\gamma_{t}$ & 3.422 & 3.422 & 3.422 \\
\hline$h=1$ voltage & $40.34 \mathrm{kV}$ & $40.34 \mathrm{kV}$ & 0 \\
\hline$h=2$ voltage & 0 & $20.17 \mathrm{kV}$ & 0 \\
\hline$h=1.85$ voltage & 0 & 0 & $2 \times 40 \mathrm{kV}$ \\
\hline$p_{0}$ & $1.695 \mathrm{GeV} / \mathrm{c}$ & $1.695 \mathrm{GeV} / \mathrm{c}$ & $1.695 \mathrm{GeV} / \mathrm{c}$ \\
\hline$\sigma(\delta)(\mathrm{LINAC})$ & 0.0029 & 0.0030 & 0.0030 \\
\hline$T_{L}$ & $400 \mathrm{~ns}$ & $400 \mathrm{~ns}$ & $400 \mathrm{~ns}$ \\
\hline$N_{\text {ext }}$ & $1 \times 10^{14}$ & $1 \times 10^{14}$ & $1 \times 10^{14}$ \\
\hline$-L \omega_{0}=Z_{s c} / n$ & $120 \Omega$ & $120 \Omega$ & $120 \Omega$ \\
\hline$R$ & $20 \Omega$ & $20 \Omega$ & $20 \Omega$ \\
\hline$\tau_{p}$ & $30 \mathrm{~ns}$ & $30 \mathrm{~ns}$ & $30 \mathrm{~ns}$ \\
\hline$N /$ turn & 100 & 100 & 100 \\
\hline$n_{\text {turns }}$ & 1201 & 1201 & 1201 \\
\hline$\sigma(\delta)(\mathrm{RING})$ & 0.0036 & 0.0030 & 0.0028 \\
\hline$I_{\text {peak }}$ & $55 \mathrm{~A}$ & $46 \mathrm{~A}$ & $35 \mathrm{~A}$ \\
\hline & & &
\end{tabular}

Table 2: Simulation parameters 


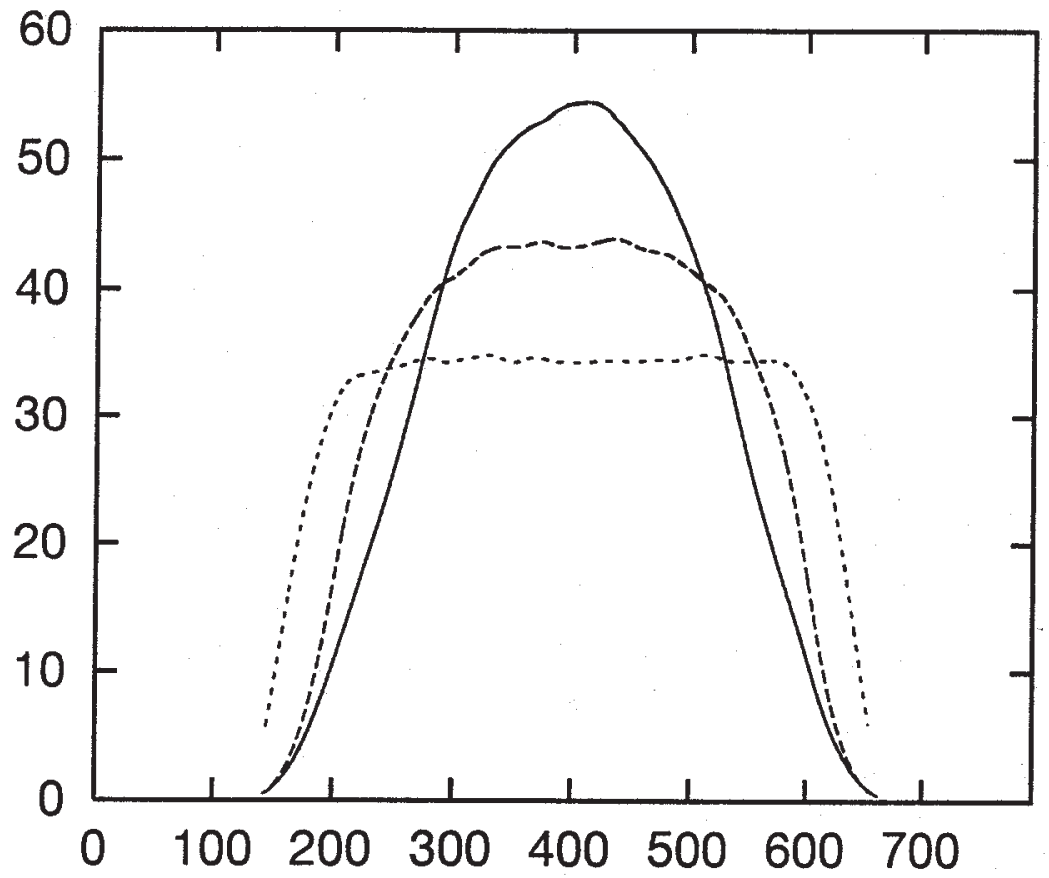

Figure 2: $I(t)(\mathrm{A})$ versus time (ns) for three rf options: $h=1$, solid line; $h=1$ and $h=2$, long dash; barrier cavity, short dash.

area to the bucket area is fixed by the length of the bunch and will not change if the voltage and momentum spread are increased appropriately.

To study these options more closely, simulations of the injection process have been made. These simulations involved only the longitudinal variables, but did include space charge and a simple model of the ring impedance. The code uses standard algorithms for everything but the impedance related forces which were handled using a new, fast, particle-particle algorithm. In this part of the algorithm the impedance induced voltage is of the form $V(t)=-R I(t)-L d I / d t$ and the current is given by

$$
I(t)=\sum_{k=1}^{N_{p}} \frac{Q}{\tau_{p}}\left(1+4\left|t-t_{k}\right| / \tau_{p}\right) \exp \left(-4\left|t-t_{k}\right| / \tau_{p}\right),
$$

where $t_{k}$ is the arrival time of the $k$ th macro particle, $Q=e N_{\text {ext }} /\left(n_{\text {turns }} N /\right.$ turn $)$ is the charge of a macro particle, and $\tau_{p}$ is the equivalent duration of a macro-particle. Table 2 lists the simulation parameters. In table 2: $p_{0}$ is the central momentum out of the LINAC which is equal to the momentum of a synchronous particle, $\sigma(\delta)$ is the standard deviation of $\delta=\left(p-p_{0}\right) / p_{0}, T_{L}$ is the chopper gate width, $N_{e x t}$ is the number of protons at extraction, $Z_{s c}$ is the space charge impedance, $R$ is the resistance associated with the ring impedance, $N /$ turn is the number of macro-particles injected each turn, $n_{\text {turns }}$ is the number of turns simulated, and $I_{\text {peak }}$ is the peak current at extraction. Note that $\sigma(\delta)$ (RING) $<\sigma(\delta)($ LINAC) for the barrier cavity. This is real and is caused by the reduced momentum deviation that occurs during reflection in the barrier.

Figure 2 shows the current pulse at extraction for the three rf options. Note that the simulated barrier cavity voltage was $2 \times 40 \mathrm{kV}$ which can be achieved with two rf cavities. 


\section{Relative Merit of the Contending rf Systems}

From the point of view of beam dynamics the single frequency, dual frequency and barrier cavity rf systems display a monotonic trend in two important quantities; bunching factor and momentum spread. The bunching factor is important in the maximum space charge tune shift

$$
\Delta \nu_{s c}=\frac{N R r_{p}}{2 \pi \nu B_{f} \beta^{2} \gamma^{3} a^{2}}
$$

where $N$ is the number of protons in the ring, $r_{p}$ is the classical proton radius, $a$ is the radius of a uniform equivalent beam, $R$ is the machine radius, and $\nu$ in the bare betatron tune. For $a=3 \mathrm{~cm}$ the tune shifts are $0.20,0.17$, and 0.13 for case 1 , case 2 , and the barrier cavity, respectively.

Other important considerations are the thresholds and growth rates of coherent instabilities. These quantities are notoriously difficult to calculate with any degree of confidence, since they involve complex coherent motion of the beam as well as the, poorly constrained, machine impedance. The simplest model is to consider the case of a coasting beam instability [5] with a current equal to the peak current of the actual bunched beam [6]. For no longitudinal instability the criterion is

$$
I_{\text {peak }}\left|\frac{Z_{\|}}{n}\right| \lesssim \frac{|\eta| \Delta E^{2}}{\beta_{0}^{2} E_{0} q}
$$

where $E_{0}=\gamma m_{p} c^{2}, \Delta E$ is the full width at half maximum energy spread, and $\eta$ is the frequency slip factor. The threshold values of $\left|Z_{\|} / n\right|$ are $130 \Omega, 110 \Omega$, and $115 \Omega$ for case 1 , case 2 , and the barrier cavity, respectively. The differences are smaller than the uncertainties, and the critical values are close to the longitudinal space charge impedance $Z_{s c} / n=120 \Omega$.

Even if the beam is unstable, there will be no problem if the growth rates are small enough. For a boxcar energy distribution, the dispersion relation for a coasting beam longitudinal mode is [7]

$$
\frac{\Omega^{2}}{\omega_{0}^{2} n^{2}}=\dot{\tau}^{2}+i \frac{q I_{\text {peak }} \eta}{2 \pi E_{0} \gamma \beta^{2}} \frac{Z_{\|}(\Omega)}{n}
$$

where $\dot{\tau}=\eta \sigma(\delta) \sqrt{\pi / 2}$. For NSNS parameters the two terms on the right hand side of equation (7) are equal for $\left|Z_{\|} / n\right| \approx 1 \mathrm{k} \Omega$. Additionally, the main contribution to the impedance is space charge which tends to reduce growth rates. Therefore,

$$
\begin{aligned}
\Omega & =\omega_{0} n \dot{\tau}\left(1+\frac{i}{\dot{\tau}^{2}} \frac{q I_{\text {peak }} \eta}{2 \pi E_{0} \gamma \beta^{2}} \frac{Z_{\| l}(\Omega)}{n}\right)^{1 / 2} \\
& \approx \omega_{0} n \dot{\tau}\left(1+\frac{i}{2 \dot{\tau}^{2}} \frac{q I_{\text {peak }} \eta}{2 \pi E_{0} \gamma \beta^{2}} \frac{Z_{\| l}(\Omega)}{n}\right)
\end{aligned}
$$

which results in a growth rate

$$
\operatorname{Im}(\Omega) \approx \frac{q I_{\text {peak }} \operatorname{Re}\left(Z_{\|}\right)}{T_{0} \gamma \sqrt{2 \pi} E_{0} \sigma(\delta)}
$$

Using equation (8) and barrier cavity parameters an e-folding time of $1 / \operatorname{Im}(\omega)=1 \mathrm{~ms}$ is obtained for $\operatorname{Re}\left(Z_{\|}\right)=640 \Omega$. This is a large parasitic resistance for a ring the size of the NSNS. 


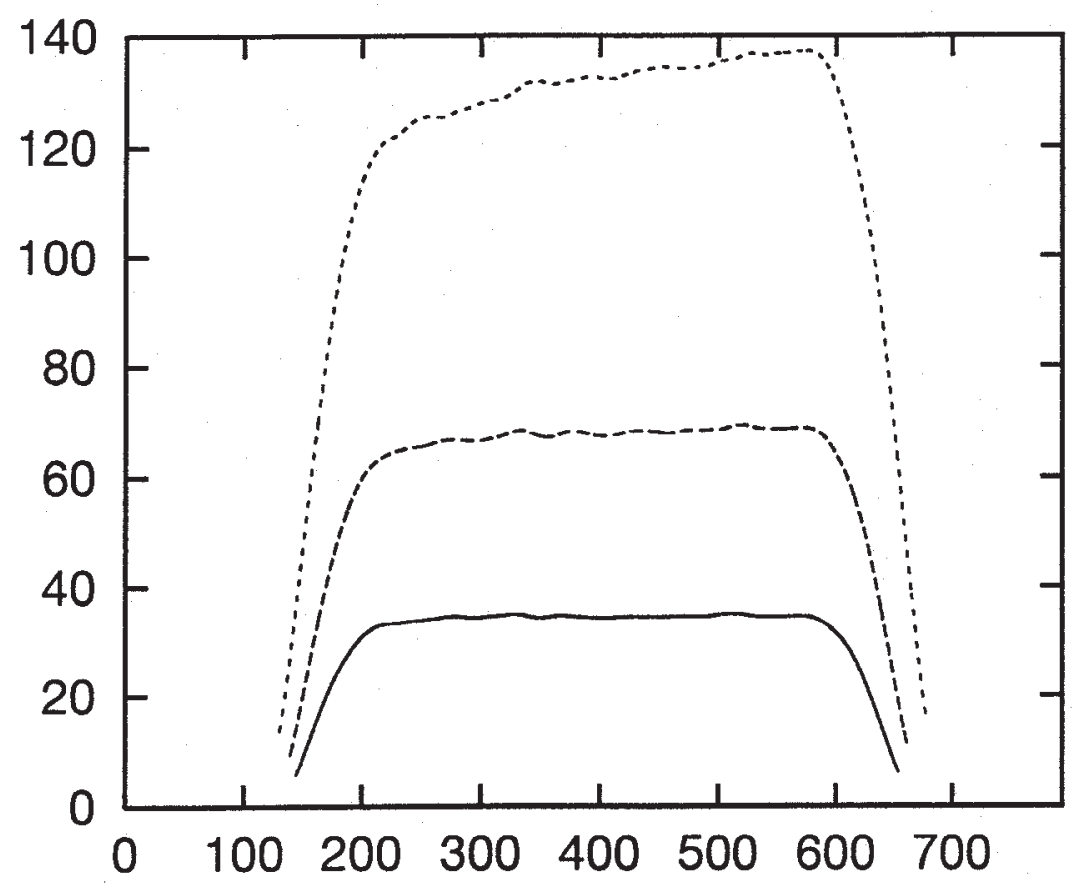

Figure 3: $I(t)(\mathrm{A})$ versus time (ns) at extraction time for a barrier cavity system with a range of beam intensities: $1 \times 10^{14}$ protons, solid line; $2 \times 10^{14}$ protons, long dash; $4 \times 10^{14}$ protons, short dash.

For transverse instabilities, a cold coasting beam approximation predicts a grow rate of $[7]$

$$
\operatorname{Im}(\Omega)=\frac{q c I_{\text {peak }} R e\left(Z_{\perp}\right)}{4 \pi E_{0} \nu} .
$$

The transverse resistive wall impedance is $Z_{\text {wall }} \approx(\operatorname{sgn}(\omega)-i) 3.3 \sqrt{\omega_{0} / \omega} \mathrm{k} \Omega / \mathrm{m}$, yielding a growth rate of $\operatorname{Im}(\Omega) \approx 400 \sqrt{\omega_{0} / \omega} \mathrm{s}^{-1}$ for a peak current of $35 \mathrm{~A}$. The shortest e-folding time of this instability occurs for $\omega=(4-\nu) \omega_{0}$ and is $1.1 \mathrm{~ms}$ which is comparable to the cycle time. Other sources of transverse impedance will be considered elsewhere [8].

Before leaving the topic of instabilities we address the reliability of using the coasting beam dispersion relations. These equations neglect the longitudinal forces created by the $\mathrm{rf}$ and, as is well known, do not give an accurate picture of phenomena with time scales $\gtrsim$ the synchrotron period. The NSNS is somewhat uncommon in this regard since the momentum spreads in Table 2 imply an rms synchrotron tune $\nu_{s} \approx|\eta| C \sigma(\delta) / 2 \pi \sigma(\ell)=5 \times 10^{-4}$. The entire cycle is less than a synchrotron period, so synchrotron side band and synchrotron mode coupling instabilities will have e-folding times of order the cycle time and will not be important.

\section{Future Upgrades}

The viability of future intensity upgrades is addressed in Figure 3 . In this figure, current pulses at extraction time for the barrier cavity system with $1 \times 10^{14}, 2 \times 10^{14}$, and $4 \times 10^{14}$ extracted protons per pulse are shown. All other input parameters are identical to column 3 in Table 2. In particular, the cavity voltage was kept at $2 \times 40 \mathrm{kV} /$ turn and the accumulation time was kept at 1201 turns. The increase in bunch length with intensity is due mainly to the space charge impedance. Figure 4 shows the voltages due to $\mathrm{rf}$, space charge, and machine resistance for $4 \times 10^{14}$ protons at extraction 


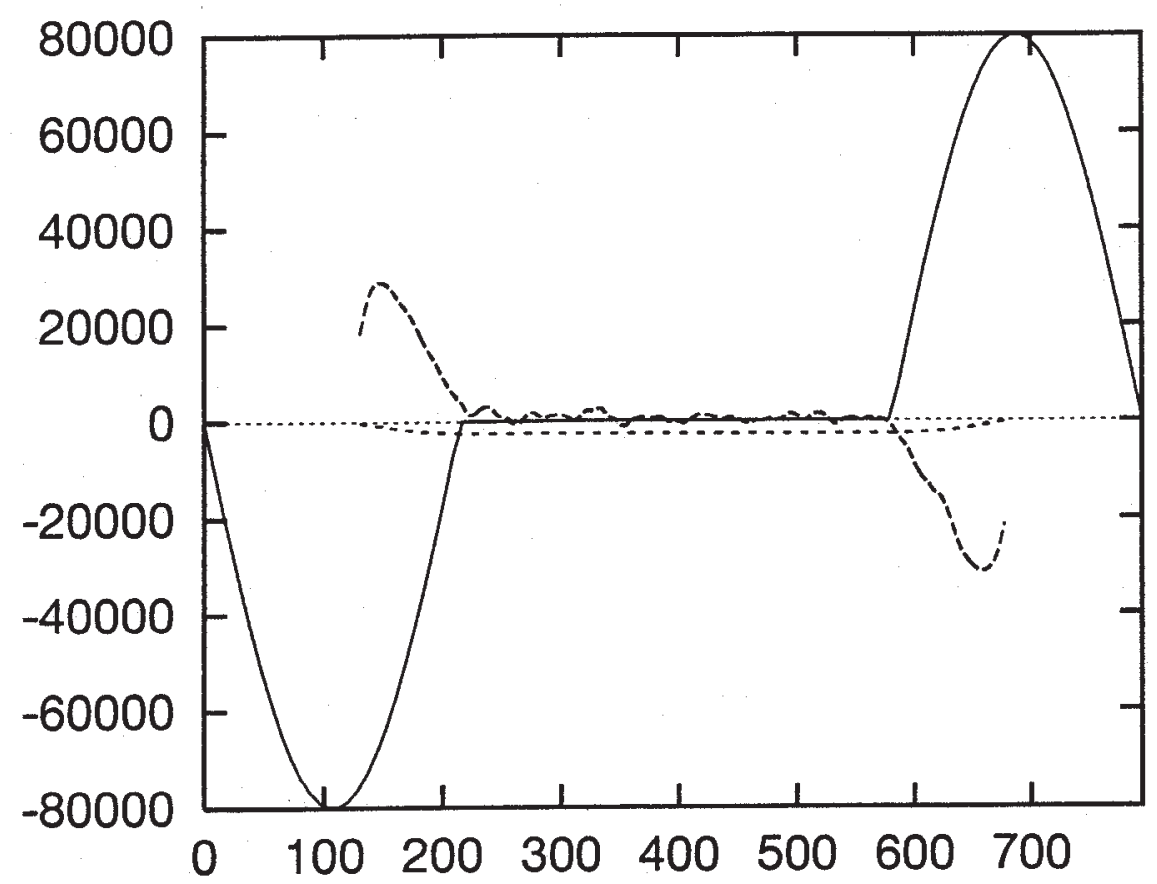

Figure 4: voltage components (V) versus time (ns) at extraction time for $4 \times 10^{14}$ protons in a barrier cavity system: solid line, rf voltage; long dash, space charge induced voltage; short dash, voltage due to machine resistance. The machine impedance was taken to be $Z(n)=(20+i n 120) \Omega$.

time. From figure 4 it is clear that no macro-particles experience the full $80 \mathrm{kV}$ available per turn, but the gap in the beam is reduced from $280 \mathrm{~ns}$ to $240 \mathrm{~ns}$.

\section{Conclusions}

All of the cases shown in Figure 2 can be realized with two, 4 meter rf cavities, so all are viable options from an engineering point of view. Additionally, the cavity resonant frequencies are all within a factor of two of each other. Hence, only a single ferrite loaded cavity needs to be designed and the differences in resonant frequency can be accomplished by varying the external capacitance across the gap. This state of affairs means that the base design could be for a barrier cavity system, and a single or dual frequency system could be easily implemented. Stability considerations suggest that two rf stations should be sufficient, but a third could be installed.

\section{References}

[1] J.E. Griffin, C. Ankenbrandt, J.A. MacLachlan, A. Moretti, IEEE TNS, 30, p. 3502, 1983.

[2] V.K. Bharadwaj, J.E. Griffin, D.J. Harding, J.A. MacLachlan, IEEE TNS, 34, p. 1025, 1987.

[3] M. Blaskiewicz, 8th ICFA Beam Dynamics Workshop, 1995.

[4] M. Blaskiewicz, J.M. Brennan 5th European Particle Accelerator Conference, 1996.

[5] A.G. Ruggiero, V.G. Vaccaro ISR-TH/68-33 (1968). 
[6] D. Boussard, CERN LABII/RF/INT/75-2 (1975).

[7] J.L. Laclare, CERN 85-19, p377, 1985.

[8] A.G. Ruggiero, M. Blaskiewicz, NSNS tech note in preparation. 